\title{
Chimney Graft Technique Combined With Embolization for Treating Ruptured Aortic Arch Lesions
}

\author{
Xianhao $\mathrm{Bao}^{1+}$, Yuxi Zhao ${ }^{1 \dagger}$, Tao $\mathrm{Li}^{2 \dagger}$, Mingwei $\mathrm{Wu}^{1}$, Zhaoxiang Zeng ${ }^{1}$, Minxin Gao ${ }^{1}$, \\ Ding $\mathrm{Xu}^{1}$, Jiaxuan Feng ${ }^{1 *}$ and Rui Feng ${ }^{1 *}$ \\ 'Department of Vascular Surgery, Shanghai General Hospital, Shanghai Jiao Tong University School of Medicine, Shanghai, \\ China, ${ }^{2}$ Department of Cardiovascular Surgery, Jinling Hospital, Medical School of Nanjing University, Nanjing, China
}

OPEN ACCESS

Edited by:

Giovanni Mariscalco, University Hospitals of Leicester NHS

Trust, United Kingdom

Reviewed by:

Maruti Haranal,

National Heart Institute, Malaysia

Shaojung $\mathrm{Li}$

Taipei Muncipal Wan Fang

Hospital, Taiwan

${ }^{*}$ Correspondence:

Rui Feng

webmaster@xueguan.net

Jiaxuan Feng

fengjiaxuan01@163.com

tThese authors have contributed equally to this work and share first

authorship

Specialty section:

This article was submitted to

Heart Surgery,

a section of the journa

Frontiers in Cardiovascular Medicine

Received: 15 June 2021 Accepted: 03 September 2021

Published: 04 October 2021

Citation

Bao X, Zhao Y, Li T, Wu M, Zeng Z, Gao $M, X u D$, Feng $J$ and Feng $R$ (2021) Chimney Graft Technique

Combined With Embolization for Treating Ruptured Aortic Arch Lesions. Front. Cardiovasc. Med. 8:711283. doi: 10.3389/fcvm.2021.711283
Background: This study aimed to share the experience in applying the chimney graft technique combined with embolization for treating aortic arch rupture under emergency conditions and evaluating early-term results in these patients.

Methods: This study retrospectively included patients with ruptured aortic arch lesions who received the chimney graft technique combined with embolization between March 2016 and March 2021. The primary endpoint was a technical success, deemed as successful stent graft deployment to the planned location, patency of the target branch vessel, and absence of significant type I endoleak. The secondary endpoint was clinical success defined with the size of false lumen in follow-up remaining unchanged or decreasing over time, 30-day mortality, complication, and primary patency of chimney graft.

Results: This study included 12 patients (age, $61 \pm 12$ years; male, 83\%). Five patients (42\%) received single chimney, one patient (8\%) received double chimney, and six patients (50\%) received triple chimney. Intraoperative type I endoleak occurred in six patients (50\%) who underwent endovascular embolization in the primary operation. Postoperative type I endoleak, evaluated by computed tomography angiography examination following the primary operation, occurred in seven patients (58\%), including one patient who received endovascular embolization two times. All patients with post-operative type I endoleak were successfully re-treated using coil and Onyx glue within 1 week, and the median length of stay was $22 \pm 11$ days (range: $7-44$ days). Overall technical success was $100 \%$. Eleven patients had completed their follow-up (median, 12 months, range: 1-34 months), and one patient was out of contact. The 30-day mortality was 9\% (1/11, post-operative death of a patient with cerebral hemorrhage). No major complications and no chimney compression, migration, occlusion, or stenosis were recorded during followup. Seven patients (58\%) have $\geq 6$ months of clinical follow-up time with appropriate imaging. In four (57\%) of these patients, diameter stabilization was detected, whereas three (43\%) experienced significant reduction ( $\geq 5 \mathrm{~mm}$ ).

Conclusion: The patients in this study had satisfactory early-term outcomes. The chimney graft technique combined with coil and Onyx glue embolization may be a safe and effective treatment for ruptured aortic arch lesions under emergency conditions.

Keywords: chimney graft, embolization, ruptured aortic arch lesions, endoleak, endovascular treatment 


\section{INTRODUCTION}

Aortic arch rupture is an extremely rare but potentially fatal condition. Open surgery remains the standard strategy for this emergency. However, some patients with advanced age and/or multiple organ dysfunction cannot tolerate the traditional open repair (1-3). Due to limited proximal anchoring area for ruptured aortic arch lesions, hybrid technique is safe and reliable in dealing with arch pathologies. However, it still had certain operative trauma because of original anatomical structure damage $(4,5)$. Thoracic endovascular aortic repair (TEVAR) has been widely used to apply innovative techniques or remodeled stent grafts, including fenestrated grafts, scallop stent grafts, branch grafts, and chimney grafts (CGs) (6-8). Although this approach is minimally invasive, it usually requires customization for each patient due to the complexity and variability of the superior branch of the aortic arch.

In the CG technique, standardized and ready-made stent grafts are used, allowing for emergency treatment of aortic rupture lesions with insufficient sealing zones (9). CGs extend the sealing zone of thoracic aortic stent graft to a certain extent and ensure blood flow to vital branch arteries (10-12). Therefore, CG technique can be an urgent choice for patients with serious complications unsuitable for open surgery $(13,14)$. However, due to a lack of customized stent grafts and technical guidelines, the result of CG technology is not always satisfactory. According to reports, the occurrence of type I endoleak (EL-I) following chimney-TEVAR (cTEVAR) can be as high as $18 \%-50 \%$ (1518). For ease of description, the sac outside the aortic stent graft is temporarily defined as a false lumen (FL). The gutter between aortic stent graft and CGs might allow blood to enter FL, leaving the risk of rupture or bleeding of aortic arch unsettled. The deployment of coil and Onyx glue to induce thrombosis in FL and gutters based on the CG technique is a key strategy to solve this problem, which is feasible and effective based on our experience in treating aortic arch rupture patients $(19,20)$.

\section{MATERIALS AND METHODS}

\section{Patients}

Between March 2016 and March 2021, our center treated 34 patients with ruptured aortic arch lesions. The inclusion criteria for this study were as follows: (1) ruptured aortic arch lesions were diagnosed using computed tomography angiography (CTA) or digital subtraction angiography (DSA); (2) first onset, or was treated with open surgery or TEVAR, but the treatment failed; (3) CG and embolization techniques were used in the hospitalization process. Exclusion criteria were as follows: (1) intramural hematoma and aortic penetrating ulcer; (2) reconstruction of superior branches of aortic arch through cervical or intrathoracic vascular bypass combined with TEVAR; (3) during operation, other TEVAR techniques such as in situ fenestration, slot, and branch were used; (4) both techniques described in this study were applied in operation but without thrombosis of FL or gutters. A total of 12 patients who underwent therapeutic strategy was included in the present analysis gradually. The study protocol was approved by the local Institutional Review Board and Ethics
Committee. All patients enrolled in the study provided informed consent for the procedure.

\section{Selection of Endovascular Prosthesis}

The size and length of stent grafts used in each case were determined by information acquired from CTA and intraoperative aortography with calibrated catheters. The selection of CG type, aortic stent graft type, and embolic material was based on institutional practice and surgeon's preference, as were other technical aspects of TEVAR procedures performed. There were no device exclusions.

\section{The First Operation Procedure}

All patients received general endotracheal anesthesia. The unilateral femoral artery was exposed through a groin oblique incision, and a 6-French catheter sheath was cannulated, from which a 5-French pigtail catheter was put into ascending aortic artery via a 0.035 -inch guidewire before systemic heparinization. The aortic stent graft was introduced upward into the thoracic aortic arch via femoral artery access. The other side of femoral artery was exposed by the described method, and a catheter was preset in FL.

By taking triple chimney (TC) as an example, bilateral brachial arteries and left common carotid artery (LCCA) were exposed through an upper arm longitudinal incision and cannulated with an 8-French catheter sheath in patients who received TC. Three CGs were preset by introducing a guidewire into the ascending aorta, from the left subclavian artery (LSA), LCCA, and innominate artery (IA). Aortic arch angiography was performed to confirm the crevasse position, the branches of aortic arch, and deployment position.

Under controlled hypotension (systolic blood pressure $<90$ $\mathrm{mmHg}$ ) and fluoroscopy, the aortic stent graft was first deployed, followed by CGs. Thus, the ischemic time of superior branch of aortic arch was minimized. All proximal CGs beyond the tectorial membrane part of aortic stent graft range $\sim 5-10 \mathrm{~mm}$, whereas the distal part of CGs was deployed in respective branch arteries (Figures 1A,B). If intraoperative EL-I was found by aortography, embolic materials were immediately filled in FL or gutters between aortic stent graft and CGs through a pre-road catheter. Complete aortography confirmed the patency of arch branches and the elimination of FL without evidence of endoleak.

\section{The Second Operation Procedure}

Patients who complained of chest pain after the initial operation were reexamined by CTA, which revealed post-operative EL-I (Figure 1C). All patients underwent reintervention to embolize lesions and gutters. Local anesthesia was performed. The right femoral artery or the left or right brachial artery was chosen as the access artery, according to anatomic characteristics of the entry location. We took a right femoral artery as an example. After the percutaneous puncture, a 5-French pigtail catheter cannulated with a 6-French catheter sheath was placed into the ascending aortic artery via a 0.035 guidewire. The guidewire and catheter were promoted along the curve formed between the ascending aorta and the aortic valve and inserted into the lesions of the aortic arch or FL through selecting the gutters after the reversal 

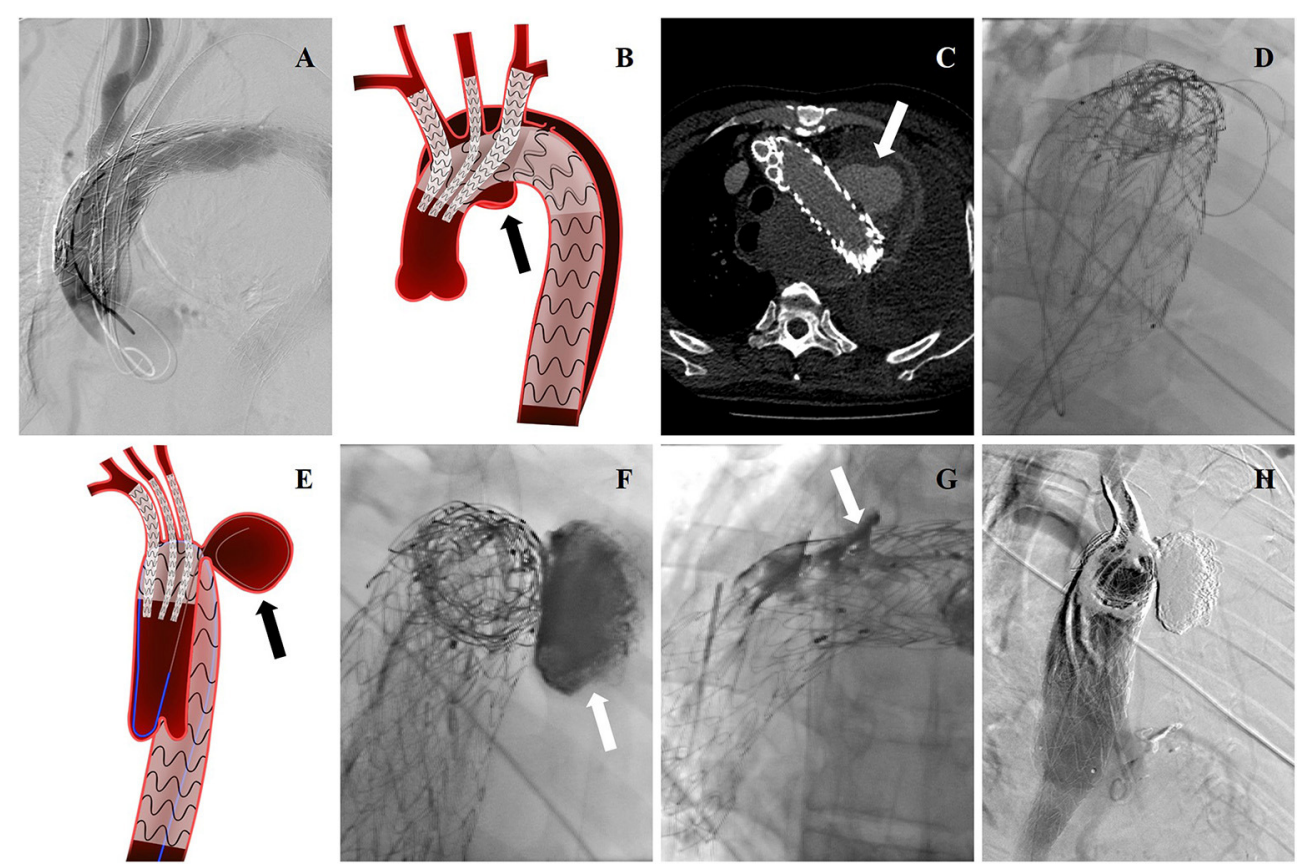

FIGURE 1 | (A) Intraoperative DSA showing this patient was treated with TEVAR before, and a new pseudoaneurysm occurred. cTEVAR was performed in the first operation. (B) Endovascular treatment was shown by cartoon graph. (C) Post-operative EL-I was revealed by CTA reexamination, and pseudoaneurysm (arrow) almost occupied the entire left thoracic cavity. (D) The guidewire and catheter entered into FL through gutters by choosing femoral artery as the access artery in the second operation. (E) The cartoon graph demonstrated the method of selecting guidewire into FL (arrow). (F) Onyx glues were injected into FL through a microcatheter (arrow). (G) Onyx glues were injected into gutters (arrow). (H) Endoleak disappeared in the last aortography.

(Figures 1D,E). Detachable coils were deployed into FL, where thrombosis was induced, filling the whole space. A microcatheter (Echelon-10; ev3 Neurovascular, Irvine, Calif) was introduced, through which Onyx glues (ev3 Neurovascular) were injected into FL and gutters (Figures 1F,G). The volume of injected Onyx glue was estimated according to anatomic parameters. Glue injection was stopped once gutters were fully filled, which could be observed through angiography. After injection, a final aortography was performed to confirm the patency of arch branches and elimination of EL-I (Figure 1H).

\section{Data Collection and Analysis}

Patient demographics, characteristics of pathologies, type of stent grafts, type of embolic materials, and individual procedural details were all collected and reviewed. Three-dimensional reconstructions of pre-operative and post-operative CTA were processed by TERARECON workstation (Aquarius, CA). All measurements of aorta and branch arteries were conducted using central line model.

\section{Endpoints and Follow-Up}

The primary endpoint was a technical success, which was deemed as successful deployment of stent graft to the planned location, patency of the target branch vessel, and absence of significant ELI. The secondary endpoint was a clinical success with the size of false lumen in follow-up remaining unchanged or decreased, 30-day mortality, complication, and primary patency of the CG.
Physical examination, CTA scans, and 3D reconstruction were performed at 3 months, 6 months, and then annually after a second operation. The morphology, position, and patency of aortic grafts and CGs were evaluated. Thrombosis of FL or gutters was analyzed. Additional CTA examinations were obtained in patients manifesting signs or symptoms of adverse reactions. All evaluations were made by vascular surgeons and were verified independently by the first author and the coauthor.

\section{Statistical Analysis}

All analyses were conducted using IBM SPSS 19.0 software (IBM Corp., Armonk, NY). Continuous variables in Gaussian distribution were described as mean \pm standard deviations (SD). Skewed variables were summarized as median and range. The specific data were provided as the count and percentage. The diameters were compared using two-sided paired sample Student's $t$-tests. A $p$-value $<0.05$ was considered statistically significant.

\section{RESULTS}

\section{Pre-operative Characteristics of Patients}

Under emergency conditions, 12 patients were treated with the CG technique in combination with embolization. Patients were diagnosed as ruptured type $\mathrm{B}$ aortic dissection in nine patients (75\%), ruptured aortic arch aneurysm in two patients (17\%), and traumatic transection of the aorta in one patient $(8 \%)$. The 
percentage of endovascular procedures was 35\% (12/34) for all patients with ruptured aortic arch lesions treated in our hospital. Table 1 lists the demographic data, risks for open surgery, and clinical characteristics of 12 patients receiving endovascular repair. The mean age of patients was $61 \pm 12$ years (range: $41-$ 83 years). Nine patients with severe complications or previous

TABLE 1 | Patient characteristics.

\begin{tabular}{|c|c|}
\hline Characteristics & $n / N(\%)$ \\
\hline Male sex & 10/12 (83) \\
\hline Age, years, mean $\pm S D$ & $61 \pm 12$ \\
\hline Hypertension & 10/12 (83) \\
\hline Smoking & 3/12 (25) \\
\hline \multicolumn{2}{|l|}{ Clinical symptoms } \\
\hline Chest pain & 7/12 (58) \\
\hline Hemoptysis & $2 / 12(17)$ \\
\hline Dyspnea & $3 / 12(25)$ \\
\hline Hoarseness & $3 / 12(25)$ \\
\hline Others* & $1 / 12(8)$ \\
\hline \multicolumn{2}{|l|}{ Risk of open surgery } \\
\hline Advanced age & $3 / 12(25)$ \\
\hline Severe COPD & 1/12 (8) \\
\hline Ischemic stroke & $3 / 12(25)$ \\
\hline Secondary infection & $2 / 12(17)$ \\
\hline ITP & $1 / 12(8)$ \\
\hline \multicolumn{2}{|l|}{ Previous aortic repair } \\
\hline TEVAR & 2/12 (17) \\
\hline Open repair & 2/12 (17) \\
\hline
\end{tabular}

surgical history were considered a high surgical risk and treated with endovascular procedures.

\section{Operative Characteristics}

The treatment strategy according to entry location is presented in Table 2. The proximal ends of entry tears were $<15 \mathrm{~mm}$ away from LSA lateral margin in eight patients (67\%), were $<15 \mathrm{~mm}$ away from LCCA lateral margin in three patients $(25 \%)$, and were $<15 \mathrm{~mm}$ away from IA lateral margin in one patient $(8 \%)$. Single-chimney (SC) technique was performed in five $(5 / 12,42 \%)$ patients; CGs were deployed to LSA in two $(2 / 5,40 \%)$ patients and LCCA in three $(3 / 5,60 \%)$ patients. Double-chimney (DC) technique was performed in one $(1 / 12,8 \%)$ patient, in which CGs were deployed to LCCA and LSA. Six (6/12, 50\%) patients received TC in IA, LSA, and LCCA. All CGs were parallel and along the direction of blood flow.

\section{Stent Grafts and Embolic Materials Data}

The intraoperative details of the two operations are presented in Tables 3, 4. The aortic stent grafts used included those by TAG (W. L. Gore \& Associates, AZ, USA; $n=7$ ), Valiant (Medtronic, Minneapolis, MN, USA; $n=4$ ), and Zenith (Cook, Bloomington, IN, USA; $n=1)$. The mean oversizing of main stents was $116.4 \pm$ $5.4 \%$. In total, 12 patients were implanted with 29 CGs, included those by Viabahn (W. L. Gore \& Associates, AZ, USA; $n=14$ ), Sinus (Optimed, Ettlingen, Germany; $n=10$ ), Fluency (C.R. Bard, Tempe, AZ, USA; $n=4$ ), and Everflex (ev3 Endovascular Inc., Minn, USA; $n=1)$. CGs include covered stents and baremetal stents (Table 3). Bare stents were used in two cases (17\%), covered stents were used in nine cases $(75 \%)$, and both stents were used in one case ( $8 \%)$.

A total of 108 coils were deployed in 10 patients (83\%). Between 4 and 57 coils (median, $12 \pm 17$ ) were used in each patient. Five patients (50\%) used coils in the first operation, in which the embolism area was filled up after coils were

TABLE 2 | Treatment strategy according to the location of entry.

\begin{tabular}{|c|c|c|c|c|c|c|c|c|c|c|c|c|}
\hline \multirow[t]{3}{*}{$N$} & \multirow[t]{3}{*}{ Location of entry } & \multicolumn{6}{|c|}{ First operation } & \multicolumn{4}{|c|}{ Second operation } & \\
\hline & & \multicolumn{3}{|c|}{ Number of CGs } & \multicolumn{2}{|c|}{ FL } & \multicolumn{2}{|c|}{ Gutter } & \multicolumn{2}{|c|}{ FL } & \multicolumn{2}{|c|}{ Gutter } \\
\hline & & IA & LCCA & LSA & C & $\mathbf{G}$ & C & $\mathbf{G}$ & C & $\mathbf{G}$ & C & G \\
\hline 1 & $\mathrm{Z1}$ & 1 & 1 & 1 & / & / & / & / & 2 & / & 2 & 1 \\
\hline 2 & $\mathrm{Z} 2$ & 1 & 1 & 1 & / & / & / & / & 4 & / & 2 & / \\
\hline 3 & Z2 & 1 & 2 & 1 & / & / & / & / & 54 & 10 & 3 & 2 \\
\hline 4 & $\mathrm{Z} 2$ & / & 1 & / & 6 & / & 1 & / & / & / & / & / \\
\hline 5 & $\mathrm{Z3}$ & / & / & 1 & / & / & / & / & / & 2 & / & 2 \\
\hline 6 & Z3 & 1 & 1 & 1 & / & / & / & / & 5 & 1 & / & 1 \\
\hline 7 & Z3 & / & 1 & I & 2 & / & 1 & / & 9 & / & / & I \\
\hline 8 & Z3 & 1 & 1 & 1 & / & / & / & / & / & 8 & / & 2 \\
\hline 9 & Z3 & / & 1 & 1 & 5 & 1 & / & 1 & / & / & / & I \\
\hline 10 & Z3 & / & / & 2 & 4 & 4 & / & 1 & / & / & / & / \\
\hline 11 & Z3 & 2 & 2 & 1 & / & 6 & / & 2 & 2 & / & 2 & I \\
\hline 12 & Z3 & / & 1 & / & 4 & 2 & / & 1 & / & / & / & / \\
\hline
\end{tabular}

C, number of coils; G, volume of Onyx glue. 
TABLE 3 | Characteristics of aortic and chimney stent grafts.

\begin{tabular}{lc}
\hline Aortic and Chimney Stent grafts & N (\%) \\
\hline Aortic stent grafts & \\
TAG (W. L. Gore \& Associates, AZ, USA) & $7(58)$ \\
Valiant (Medtronic, Minneapolis, MN, USA) & $4(33)$ \\
Zenith (Cook, Bloomington, IN, USA) & $1(8)$ \\
Chimney grafts & \\
Covered stent & \\
Viabahn (W. L. Gore \& Associates, AZ, USA) & $14(48)$ \\
Sinus (Optimed, Ettlingen, Germany) & $7(25)$ \\
Fluency (C.R. Bard, Tempe, AZ, USA) & $4(14)$ \\
Bare-metal stent & \\
Sinus (Optimed, Ettlingen, Germany) & $3(10)$ \\
Everflex (ev3 Endovascular Inc., Minn, USA) & $1(3)$ \\
\hline
\end{tabular}

TABLE 4 | The choice of access artery for embolization.

\begin{tabular}{ll}
\hline Access artery & $\mathbf{n / N}(\%)$ \\
\hline The first operation $(\mathbf{N}=\mathbf{6})$ & \\
Right femoral artery & $2 / 6(33)$ \\
Left femoral artery & $2 / 6(33)$ \\
Left brachial artery & $2 / 6(33)$ \\
The second operation $(\mathbf{N}=\mathbf{8})$ & \\
Right femoral artery & $4 / 8(50)$ \\
Left brachial artery & $3 / 8(38)$ \\
Right brachial artery & $1 / 8(13)$ \\
\hline
\end{tabular}

released one by one through a pre-road catheter. Six patients $(60 \%)$ utilized coils in the second operation, during which coils were deployed through the catheter, which was selected into FL or gutters. One patient (10\%) used coils in both operations. Interlock (Boston Scientific, Natick, MA, USA; $n=43$ ) was most frequently used (in eight patients, 80\%), followed by MWCE (Cook Medical, Bloomington, IN, USA; $n=65$ ) in two patients (20\%), in one of whom required 57 MWCE coils. Nine patients (75\%) underwent Onyx (ev3, Irvine, California, USA) glue injection, four $(44 \%)$ in the first operation and five $(56 \%)$ in the second operation; the mean volume of glue injected was $5 \pm 4 \mathrm{ml}$ (range: 1-12). The total injection volume of Onyx was $47 \mathrm{ml}$.

\section{Post-operative Outcomes and Follow-Up}

All stent grafts and embolic materials were successfully deployed. Complete thrombosis of FL of the aortic arch was noted in all 12 patients. The treatment of three representative patients is showed separately in Figures 1-3. The mean interval from onset to the first operation was $2 \pm 1$ days (range: $1-7$ days) in 12 patients. The time between the first surgery and revision was 3 \pm 1 day (range: $2-7$ days) in six patients. The mean endovascular procedure time of the first operation was $224 \pm 87 \mathrm{~min}$ (range: 105-370 $\mathrm{min}$ ), and that of the second operation was $179 \pm 95 \mathrm{~min}$ (range: 40-360 $\mathrm{min}$ ). All patients entered ICU after surgery for monitoring. The mean time of ICU stay was $4 \pm 7$ days (range:

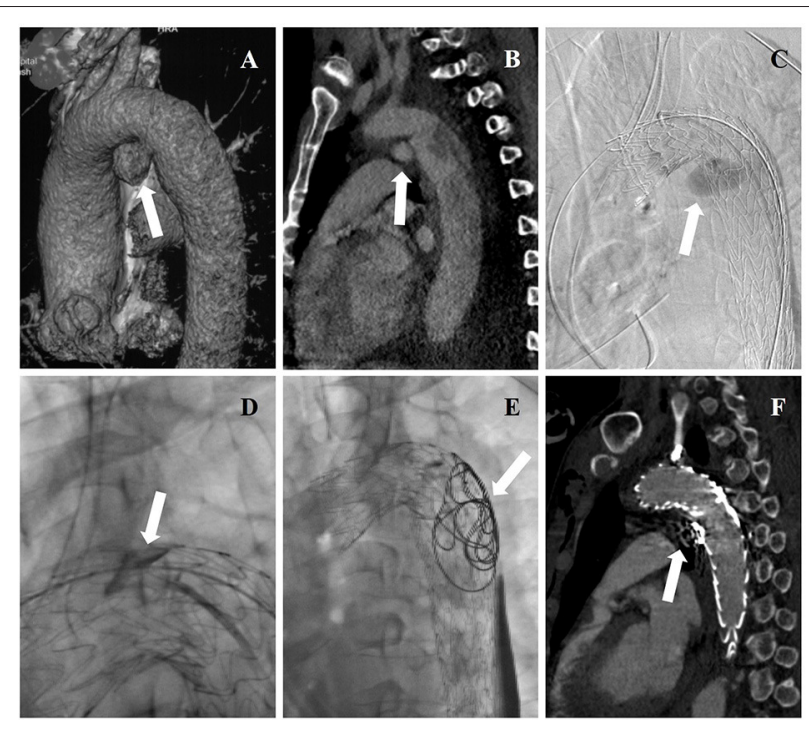

FIGURE 2 | (A) Three-dimensional reconstructions of pre-operative CTA showing concomitant aortic arch pseudoaneurysm (arrow) of traumatic aortic dissection. (B) Pre-operative CTA showing the entry tear (arrow) of aortic dissection. (C) Intraoperative DSA showing deployment of aortic stent graft and CGs, and angiography through a pre-road catheter to FL revealed intraoperative EL-I (arrow). (D) Onyx glues were injected into gutters (arrow) through a microcatheter. (E) Coils and Onyx glues were deployed into FL (arrow) through a pre-road catheter. (F) The aortic dissection and pseudoaneurysm were entirely excluded while endoleak disappeared (arrow) in post-operative CTA.

1-25 days). All patients completed cTEVAR and embolization during one hospitalization. The average hospitalization time was $22 \pm 11$ days (range: 7-44 days). The mean in-hospital expense was $\$ 65,695 \pm 21,625$ (range: $\$ 26,419-\$ 95,828$ ). Two patients (17\%) experienced brain lacunar infarction during hospitalization and were cured before discharge.

Eleven patients (92\%) completed the follow-up. They were closely followed up for a median of 12 months (range: 1-34 months). One patient (8\%) was out of contact immediately after discharge, and his status was unknown. In this cohort, no stent graft migration, component separation, or fracture occurred. During the follow-up period, no cerebral infarction, no new endoleak, and no CG stenosis or occlusion were observed. One patient with severe thrombocytopenia eventually died of a cerebral hemorrhage on the 18th day after the second operation in another hospital, leading to a $9 \%(1 / 11) 30$-day mortality. Seven patients (58\%) have $\geq 6$ months of clinical follow-up time with appropriate imaging follow-up. In four (57\%) of these patients, diameter stabilization was detected, whereas three (43\%) experienced a significant reduction $(\geq 5 \mathrm{~mm})$.

\section{DISCUSSION}

Aortic arch lesions present a challenge and are at the forefront of aortic disease treatment due to their involvement of superior branch arteries $(11,14,21-23)$. When aortic dissection, aortic aneurysm, trauma, or other injury factors cause rupture of aortic 


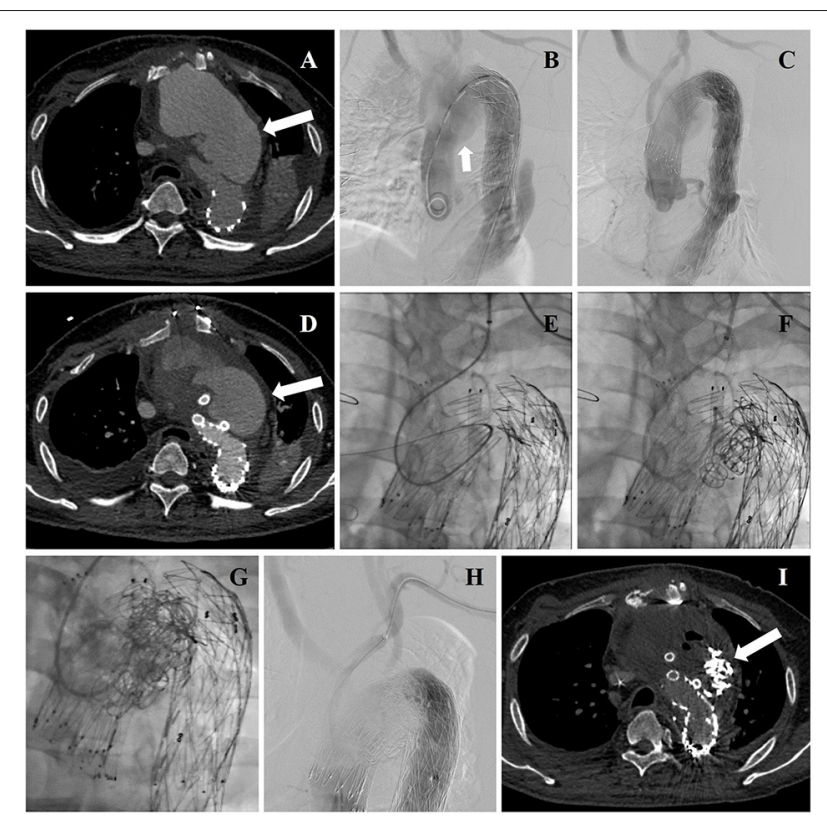

FIGURE 3 | (A) Aortic arch pseudoaneurysm (arrow) was found after TEVAR by CTA admission. (B) Intraoperative DSA identified the location of lesion again (arrow). (C) Intraoperative aortography showing deployment of aortic stent graft and CGs. (D) CTA reexamination revealed post-operative EL-I (arrow). (E) The guidewire and catheter entered into FL through gutters by choosing left brachial artery as the access artery in the second operation. (F) Coils were deployed into FL and gutters. (G) Onyx glues were deployed into FL and gutters through a microcatheter. (H) A final aortography confirmed the patency of arch branches and the exclusion of EL-I. (I) CTA examination was performed 3 months after the second operation showing that endoleak disappeared (arrow).

arch lesions, it will progress rapidly and adversely affect heart and lung function, making it one of the most critical diseases with the highest mortality rate $(8,20)$.

Most ruptured aortic arch lesions are associated with circulatory instability, and some lesions are secondary to open or endovascular procedures $(2,3,16)$. As a result, open surgery is often difficult to perform due to its invasiveness or poor pre-operative condition of patients. Furthermore, patients who received open surgery had high mortality and a high incidence of complications $(1,20)$. In comparison, stent grafting is a minimally invasive surgery compared to artificial blood vessel replacement during thoracotomy (17-24). Stent grafting has been recently reported in treating ruptured aortic arch lesions. However, traditional endovascular treatment cannot reconstruct arch branches while settling aortic arch lesions. The treatment of ruptured aortic arch under emergency conditions remains ineffective $(13,20)$.

How to use endovascular treatment to solve this problem becomes an important subject that requires additional research. Branched stent grafts cannot be applied to rescue ruptured aortic arch disease in emergency circumstances, as they must be customized to the arch's anatomy $(25,26)$. Similarly, fenestrated stent grafts, pre-fenestration technique, or in situ fenestration technique, which are complex and require custom-made devices, have rarely been reported in recent years $(5-9,25)$. Although the CG technique has been extensively published in treating aortic arch lesions, it is difficult to apply in ruptured aortic arch lesions due to the risk of endoleak $(10,16,20,27,28)$. However, the CG technique is easy to operate and does not require customizing stent grafts under emergency conditions, making it an excellent option for treating aortic arch rupture $(11,15,24)$. Therefore, how to properly handle post-operative endoleak of cTEVAR becomes the focus of endovascular treatment for ruptured aortic arch lesions.

Improved coil material and design makes coil embolization a very safe procedure. Extensive literature revealed that coil embolization was employed in the endovascular treatment of many types of aortic diseases $(29,30)$. Most reported cases involved remedial or assisted therapeutic methods to deal with a patent FL following TEVAR or surgical intervention (29).

Onyx glue, as a permanent liquid embolic agent, has also been widely used in treating many vascular conditions $(30,31)$. Previous research has indicated that using coils along with Onyx glue is a safe and efficient approach to create a durable thrombogenic environment $(32,33)$. However, applying coil or Onyx glue to eliminate intraoperative endoleak of CG technique for endovascular treatment of ruptured aortic arch lesions has not yet been reported.

The key to embolic elimination of EL-I lies in the entry of gutter. There are three effective strategies: reserved preroad catheter, superselection through FL, and superselection through the graft's proximal end. However, special attention should be paid during embolization to the occurrence and risk of ectopic embolism.

\section{CONCLUSIONS}

In patients with ruptured aortic arch lesions under emergency conditions, the CG technique extends the sealing zone of thoracic aortic stent graft to a certain extent, and a thrombogenic environment in FL and gutters is created using coils and Onyx glue to settle the problem of EL-I. The creation of this thrombosis after cTEVAR may be a safe and effective treatment strategy for aortic arch rupture. In the present study, earlyterm patient outcomes were satisfactory; however, long-term outcomes remain to be evaluated.

\section{DATA AVAILABILITY STATEMENT}

The original contributions presented in the study are included in the article/supplementary material, further inquiries can be directed to the corresponding author/s.

\section{ETHICS STATEMENT}

The studies involving human participants were reviewed and approved by the studies involving human participants were reviewed and approved by Informed Consent were obtained from all the patients and the study protocols were approved by the Ethical Review Board and Statistics Department of Shanghai 
General Hospital (No. 2016SQ271). The patients/participants provided their written informed consent to participate in this study. Written informed consent was obtained from the individual(s) for the publication of any potentially identifiable images or data included in this article.

\section{AUTHOR CONTRIBUTIONS}

$\mathrm{RF}$ and JF conceptualized and led the work. XB, YZ, and TL were contributed to the study design, obtaining funding, writing the article, and critical revision of the article. MW, ZZ,

\section{REFERENCES}

1. Okada K, Omura A, Kano H, Sakamoto T, Tanaka A. Recent advancements of total aortic arch replacement. J Thorac Cardiovasc Surg. (2012) 144:13945. doi: 10.1016/j.jtcvs.2011.08.039

2. Ouzounian M, LeMaire SA, Coselli JS. Open aortic arch repair: state-of-theart and future perspectives. Semin Thorac Cardiovasc Surg. (2013) 25:10715. doi: 10.1053/j.semtcvs.2013.07.001

3. Yohei K, Yujiro H, Yujiro I, Hirotsugu K, Yoshitsugu N, Yujiet S, et al. A case of ruptured aortic arch aneurysm successfully treated by thoracic endovascular aneurysm repair with chimney graft. Case Rep Surg. (2015) 2015:780147. doi: 10.1155/2015/780147

4. Yukitoshi S, Toru K, Kazuo S, Kei T, Tomohiko S, Takayuki S, et al. The efficacy and short-term results of hybrid thoracic endovascular repair into the ascending aorta for aortic arch pathologies. Eur J Cardio Thorac. (2014) 45:298. doi: 10.1093/ejcts/ezt391

5. Bergeron P, Mangialardi N, Costa P, Coulon P, Douillez V, Serreo E, et al. Great vessel management for endovascular exclusion of aortic arch aneurysms and dissections. Eur J Vasc Endovasc Surg. (2006) 32:3845. doi: 10.1016/j.ejvs.2005.12.023

6. Kasprzak P, Pfister K, Janotta M, Kopp R. EndoAnchor placement in thoracic and thoracoabdominal stent-grafts to repair complications of nonalignment. J Endovasc Ther. (2013) 20:471-80. doi: 10.1583/12-4125.1

7. Redlinger RE, Ahanchi SS, Panneton JM, Norfolk V. In situ laser fenestration during emergent thoracic endovascular aortic repair is an effective method for left subclavian artery revascularization. J Vasc Surg. (2013) 58:11717. doi: 10.1016/j.jvs.2013.04.045

8. Bosiers MJ, Donas KP, Mangialardi N, Torsello G, Riambau V, Criado FJ, et al. European multicenter registry for the performance of the chimney/snorkel technique in the treatment of aortic arch pathologic conditions. Ann Thorac Surg. (2016) 101:2224-30. doi: 10.1016/j.athoracsur.2015.10.112

9. Ohrlander T, Sonesson B, Ivancev K, Resch T, Dias N, Malina M. The chimney graft: a technique for preserving or rescuing aortic branch vessels in stent-graft sealing zones. J Endovasc Ther. (2008) 15:427-32. doi: 10.1583/ 07-2315.1

10. Feng R, Zhao Z, Bao J, Wei X, Liang W, Jing Z. Double-chimney technology for treating secondary type I endoleak after endovascular repair for complicated thoracic aortic dissection. J Vasc Surg. (2011) 54:2125. doi: $10.1016 /$ j.jvs.2010.11.120

11. Gehringhoff B, Torsello G, Pitoulias GA, Austermann M, Donas KP. Use of chimney grafts in aortic arch pathologies involving the supraaortic branches. J Endovasc Ther. (2011) 18:650-5. doi: 10.1583/11-3504.1

12. Voskresensky I, Scali ST, Feezor RJ, Fatima J, Giles KA, Tricarico $\mathrm{R}$, et al. Outcomes of thoracic endovascular aortic repair using aortic arch chimney stents in high-risk patients. J Vasc Surg. (2017) 66:920. doi: 10.1016/j.jvs.2016.11.063

13. Naughton PA, Park MS, Morasch MD, Rodriguez HE, Eskandari MK. Emergent repair of acute thoracic aortic catastrophes: a comparative analysis. Arch Surg. (2012) 147:243-9. doi: 10.1001/archsurg.2011.1476

14. Moulakakis KG, Mylonas SN, Dalainas I, Sfyroeras GS, Markatis F, Kotsis $\mathrm{T}$, et al. The chimney-graft technique for preserving
MG, and DX were contributed to the data collection, analysis, and interpretation. All authors contributed to the article and approved the submitted version.

\section{FUNDING}

This work was supported by the National Natural Science Foundation of China [81770476 and 81970208], the Science and Technology Innovation Plan of Shanghai Science and Technology Commission [20S31901700], and Shanghai RisingStar Program [20QA1408900].

supra-aortic branches: a review. Ann Cardiothorac Surg. (2013) 2:339-46. doi: 10.3978/j.issn.2225-319X.2013.05.14

15. Sugiura K, Sonesson B, Akesson M, Björses K, Holst J, Malina M. The applicability of chimney grafts in the aortic arch. J Cardiovasc Surg. (2009) 50:475-81. Available online at: https://www.minervamedica.it/en/ journals/cardiovascular-surgery/article.php?cod=R37Y2009N04A0475

16. Rancic Z, Pfammatter T, Lachat M, Hechelhammer L, Frauenfelder T, Veith FJ, et al. Periscope graft to extend distal landing zone in ruptured thoracoabdominal aneurysms with short distal necks. J Vasc Surg. (2010) 51:1293-6. doi: 10.1016/j.jvs.2009.11.076

17. Lachat M, Veith FJ, Pfammatter T, Glenck M, Bettex D, Mayer D, et al. Chimney and periscope grafts observed over 2 years after their use to revascularize 169 renovisceral branches in 77 patients with complex aortic aneurysms. J Endovasc Ther. (2013) 20:597-605. doi: 10.1583/134372.1

18. Yang J, Xiong J, Liu X, Jia X, Zhu Y, Guo W. Endovascular chimney technique of aortic arch pathologies: a systematic review. Ann Vasc Surg. (2012) 26:101421. doi: 10.1016/j.avsg.2012.05.014

19. Bangard C, Franke M, Pfister R, Deppe AC, Matoussevitch V, Maintz D, et al. Thoracic type Ia endoleak: direct percutaneous coil embolization of the aortic arch at the blood entry site after TEVAR and double-chimney stent-grafts. Eur Radiol. (2014) 24:1430-4. doi: 10.1007/s00330-014-3143-8

20. Jabr AB, Lindblad B, Dias N, Resch T, Malina M. Efficacy and durability of the chimney graft technique in urgent and complex thoracic endovascular aortic repair. J Vasc Surg. (2015) 61:886-94. doi: 10.1016/j.jvs.20 14.11.078

21. O’Callaghan A, Mastracci TM, Greenberg RK, Eagleton MJ, Bena J, Kuramochi Y. Outcomes for supra-aortic branch vessel stenting in the treatment of thoracic aortic disease. J Vasc Surg. (2014) 60:91420. doi: 10.1016/j.jvs.2013.12.053

22. Zhu Y, Guo W, Liu X, Jia X, Xiong J, Wang L. The single-centre experience of the supra-arch chimney technique in endovascular repair of type B aortic dissections. Eur J Vasc Endovasc Surg. (2013) 45: 6338. doi: 10.1016/j.ejvs.2013.02.016

23. Shahverdyan R, Gawenda M, Brunkwall J. Triple-barrel graft as a novel strategy to preserve supra-aortic branches in arch-TEVAR procedures: clinical study and systematic review. Eur J Vasc Endovasc Surg. (2013) 45:2835. doi: 10.1016/j.ejvs.2012.09.023

24. Zou J, Jiao Y, Zhang X, Jiang J, Yang H, Ma H. Early- and mid-term results of the chimney technique in the repair of aortic arch pathologies. Cardiovasc Intervent Radiol. (2016) 39:1550-6 doi: 10.1007/s00270-0161439-6

25. Chuter TAM. Branched and fenestrated stent grafts for endovascular repair of thoracic aortic aneurysms. J Vasc Surg. (2006) 43:A1115. doi: 10.1016/j.jvs.2005.10.059

26. Haulon S, Greenberg RK, Spear R, Eagleton M, Abraham C, Lioupis C, et al. Global experience with an inner branched arch endograft. J Thorac Cardiovasc Surg. (2014) 148:1709-16. doi: 10.1016/j.jtcvs.2014.02.072

27. Hiramoto JS, Schneider DB, Reilly LM, Chuter T. A double-barrel stentgraft for endovascular repair of the aortic arch. J Endovasc Ther. (2006) 13:72-6. doi: 10.1583/04-1711R.1 
28. Liu H, Shu C, Li X, Wang T, Li M, Li QM, et al. Endovascular aortic repair combined with chimney technique in the treatment of stanford type B aortic dissection involving aortic arch. Ann Vasc Surg. (2015) 29:758-63. doi: 10.1016/j.avsg.2014.12.004

29. Katayama K, Uchida N, Takahashi S, Sueda T. Scheduled re-entry coil embolization before entry coverage of thoracic endovascular stent grafting for aneurysmal chronic type B aortic dissection. Interact Cardiovasc Thorac Surg. (2012) 15:800-1. doi: 10.1093/icvts/ivs288

30. Gao X, Liang G, Li Z, Wang X, Yu C, Cao P, et al. Transarterial coil-augmented Onyx embolization for brain arteriovenous malformation: technique and experience in 22 consecutive patients. Interv Neuroradiol. (2014) 20:8390. doi: 10.15274/INR-2014-10012

31. Khaja MS, Park AW, Swee W, Evans AJ, Fritz Angle J, Turba UC, et al. Treatment of type II endoleak using Onyx with longterm imaging follow-up. Cardiovasc Intervent Radiol. (2014) 37:613-22. doi: 10.1007/s00270-013-0706-z

32. Chalouhi N, Starke RM, Tjoumakaris SI, Jabbour PM, Gonzalez LF, Hasan $\mathrm{D}$, et al. Carotid and vertebral artery sacrifice with a combination of Onyx and coils: technical note and case series. Neuroradiology. (2013) 55:9938. doi: 10.1007/s00234-013-1203-4

33. Cekirge HS, Saatci I, Geyik S, Yavuz K, Oztürk H, Pamuk G. Intrasaccular combination of metallic coils and onyx liquid embolic agent for the endovascular treatment of cerebral aneurysms. J Neurosurg. (2006) 105:70612. doi: 10.3171/jns.2006.105.5.706

Conflict of Interest: The authors declare that the research was conducted in the absence of any commercial or financial relationships that could be construed as a potential conflict of interest.

Publisher's Note: All claims expressed in this article are solely those of the authors and do not necessarily represent those of their affiliated organizations, or those of the publisher, the editors and the reviewers. Any product that may be evaluated in this article, or claim that may be made by its manufacturer, is not guaranteed or endorsed by the publisher.

Copyright (C) 2021 Bao, Zhao, Li, Wu, Zeng, Gao, Xu, Feng and Feng. This is an open-access article distributed under the terms of the Creative Commons Attribution License (CC BY). The use, distribution or reproduction in other forums is permitted, provided the original author(s) and the copyright owner(s) are credited and that the original publication in this journal is cited, in accordance with accepted academic practice. No use, distribution or reproduction is permitted which does not comply with these terms. 